\title{
A COMPARATIVE STUdY OF LSTM AND PHASED LSTM FOR GAIT PREDICTION
}

\author{
Qili Chen ${ }^{1,2,3}$, Bofan Liang ${ }^{2}$ and Jiuhe Wang ${ }^{2}$ \\ ${ }^{1}$ Faculty of Information Technology, Beijing University of Technology, Beijing, 100124, \\ China \\ ${ }^{2}$ Department of Automation, Beijing Information Science and Technology University, \\ Beijing, 100192, China \\ ${ }^{3}$ Beijing Key Laboratory of Computational Intelligence and Intelligent System, Beijing, \\ 100124, China
}

\begin{abstract}
With an aging population that continues to grow, the protection and assistance of the older persons has become a very important issue. Fallsare the main safety problems of the elderly people, so it is very important to predict the falls. In this paper, a gait prediction method is proposed based on two kinds of LSTM. Firstly, the lumbar posture of the human body is measured by the acceleration gyroscope as the gait feature, and then the gait is predicted by the LSTM network. The experimental results show that the RMSE between the gait trend predicted by the method and the actual gait trend can be reached a level of $0.06 \pm$ 0.01. And the Phased LSTM has a shorter training time. The proposed method can predict the gait trend well.
\end{abstract}

\section{KEYWORDS}

Elderly people fall, Acceleration gyro, Lumbar posture, Gait prediction, LSTM

\section{INTRODUCTION}

If the elderly people are unable to help themselves after falling, they will remain in a prone position for a long time, with after effects. In serious cases, it will threat to their lives. In the United States, for example, the older persons who are over the age of 65 fall at least once a year, but only $1 / 40$ people will be taken to the hospital, and only half of them will still be alive after a year [1]. There is some device which can detect the falls and provide an alarm, however, their functions are limited, because the fall has happened and caused the injury, despite it worked out. Even after the rescue, their trauma was restored, but the shadow left by the fall was lingering, leading to depression and other mental illness. Therefore, when the post fall detection is combined with the pre-fall prediction, the serious consequences caused by the fall can be minimized as much as possible [2].

At present, elderly person fall detection research is focused on fall detection, but there are few studies on fall prediction. However, there are not many researches on fall prediction, and most of them are based on wearable devices to predict falls. The key to fall prediction is how to improve accuracy and achieve early prediction. Many scholars have conducted related research on fall prediction: Wu used the threshold algorithm based on the horizontal and vertical speeds of the torso to predict the fall in [3], the results show that it can be predicted 300 to $400 \mathrm{~ms}$ before the fall. A.K.Bourke et al. used the threshold algorithm to perform the fall prediction in [4], fixed the acceleration sensor and the gyroscope to the chest, used the acceleration and the angular velocity to calculate the vertical velocity of the trunk, and tested the set speed threshold $-1.3 \mathrm{~m} / \mathrm{s}^{2}$ for the 
fall prediction. It achieved $100 \%$ accuracy and was able to predict a fall $322 \mathrm{~ms}$ before a torso collision or $140 \mathrm{~ms}$ before a knee collision. Nyan used a three-axis accelerometer and a two-axis gyroscope to obtain the angle of the leg and torso in [5]. Using the threshold algorithm to determine whether the leg angle exceeded the threshold $( \pm 10)$, if it exceeded, then determine the torso and leg whether the correlation coefficient exceeds the correlation coefficient threshold (0.99), and if it is exceeded, it is judged to be falling. The method achieved $95.2 \%$ sensitivity and $100 \%$ specificity, and the pre-fall time averaged $700 \mathrm{~ms}$. Li Na Tong propose a time series method to predict the fall and established a hidden Markov model (HMM) to describe the human body to assess the risk of human fall at the current moment in [6]. The output probability thresholds of acceleration time series in different motion processes are get based on the SVM, and the prediction of human fall can be realized within 200-300ms before the impact. Guangyi Shi et al. used a nine-axis inertial sensor placed at the waist and a pressure sensor placed on the sole of the foot to perform a fall prediction. It was found that the combination of the waist and the foot has higher accuracy than the use of only the waist nine-axis sensor. The accuracy of the fall prediction is $98.565 \%$. Chenchao et al. [8] used SAE and LSTM to predict the gait of lower extremity exoskeleton. According to the gait sequence of the previous period, the gait information of the next moment was effectively predicted. The average accuracy rate was over 92.9\%. ZENG $M$ [9] used convolutional neural network to recognize gait. Xu Fan et al. [10] used CNN and LSTM to classify gait and get a good prediction. Thus, it can get a good fall prediction results using sensor data, but the sequence of gait is neglected in references [2] [4], [6], and [8]. The gait recognition and prediction in references [7], [9], and [10] are carried out by using neural network, and the sequence of gait is considered, and get better accuracy. For this reason, a gait prediction method based on two kinds of LSTM and a time series prediction model is established in this paper, which is used to predict the human gait. Nine-axis accelerometer gyroscope angle sensor is used to collect gait sequence data and the LSTM network is used to predict gait sequence.

The organization of the paper is as follows. Section 1 presents the related work, analyses their progressiveness and limitations and describes the objective in this paper. Section 2 introduces LSTM neural network and phased LSTM neural network. Section 3 shows the model of gait prediction. Section 4 presents comparative experiments of prediction and shows the results. Final section concludes this paper.

\section{Tow Long And Short Time Memory NeUral Networks}

LSTM is a special type of RNN that can learn long-term dependency information. The LSTM has three gates to protect and control the state of the cells: the input gate, the forget gate, and the output gate. First, the forget gate reads the output of the previous cell $h_{t-1}$ and input of current cell $x_{t}$ and decides to discard and retain information from the cell state.

First, the forgetting gate reads the output $h_{t-1}$ and input $x_{t}$ of the previous cell and decides to discard and retain information from the cell state. Next, the input gate determines whether the value is updated or not, then a new candidate vector $\tilde{C}_{t}$ for storage state is created whose active

function is tanh function, then the cell state is updated to $C_{t}$. Finally, the output gate outputs a value. They can be calculated using equations (1) (6).LSTM structure as shown in Fig. 1 [11]

$$
\begin{gathered}
f_{t}=\sigma\left(W_{f} \cdot\left[h_{t-1}, x_{t}\right]+b_{f}\right) \\
i_{t}=\sigma\left(W_{f} \cdot\left[h_{t-1}, x_{t}\right]+b_{i}\right) \\
\tilde{C}_{t}=\tanh \left(W_{C} \cdot\left[h_{t-1}, x_{t}\right]+b_{C}\right) \\
C_{t}=f_{t} * C_{t-1}+i_{t} * \tilde{C}_{t}
\end{gathered}
$$




$$
\begin{gathered}
o_{t}=\sigma\left(W_{o} \cdot\left[h_{t-1}, x_{t}\right]+b_{o}\right) \\
h_{t}=o_{t} * \tanh \left(C_{t}\right)
\end{gathered}
$$

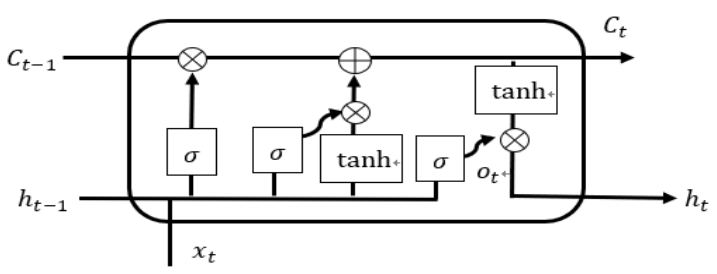

Figure 1. LSTM structure

The Phased LSTM is an improved LSTM network [12]. It can accelerate RNN or event-based sequences and improve performance. On the basis of LSTM, Phased LSTM model adds a new gate named time gate. The time gate is used for processing inputs sampled at asynchronous times. Time gate is set before forger gate, it has two phases, one is open phase, another is close phase. The open phase and close phase are controlled by $\tau$ which controls the real-time period of the oscillation, $r_{o n}$ which controls the ratio of the duration of the open phase, $s$ which controls the phase shift of the oscillation to each cell. Above three parameters can be calculated using equation (7). The time gate is calculated using equation (8).

$$
\begin{gathered}
\varphi_{t}=\frac{(t-s) \bmod \tau}{\tau} \\
k_{t}=\left\{\begin{array}{c}
\frac{2 \varphi_{t}}{r_{o n}}, \text { if } \varphi_{t}<\frac{1}{2} r_{o n} \\
2-\frac{2 \varphi_{t}}{r_{o n}}, \text { if } \frac{1}{2} r_{o n}<\varphi_{t}<r_{o n} \\
\varphi_{t}, \quad \text { otherwise }
\end{array}\right.
\end{gathered}
$$

The operation of Phased LSTM is the same as that of LSTM, except for time gate part. In LSTM, $h_{t}$ and $C_{t}$ can go through forget gate directly and be updated, but in Phased LSTM only during open phase time gate, $h_{t-1}$ and $C_{t-1}$ cansuccessfully go through forget gate and be updated. In Phased LSTM, the equations (4) and (6) should change into equations (9) and (10). The Phased LSTM structure as shown in Fig. 2.

$$
\begin{aligned}
& C_{t}=k_{t} *\left(f_{t} * C_{t-1}+i_{t} * \tilde{C}_{t}\right) \\
& +\quad\left(1-k_{t}\right) \\
& * C_{t-1} \\
& h_{t}=k_{t} *\left(o_{t} * \tanh \left(C_{t}\right)\right) \\
& +\quad\left(1-k_{t}\right) \\
& * h_{t-1}
\end{aligned}
$$

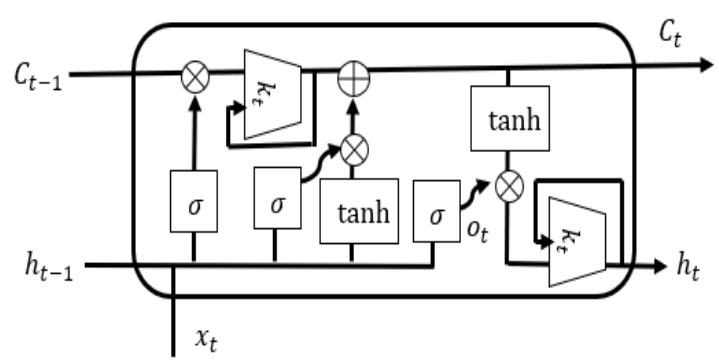

Figure 2. LSTM structure 


\section{Gait Prediction ModeL}

Two Task were study in this paper, one is single gait feature prediction of the gait, the other is multi-feature gait prediction of the gait.

For single gait feature prediction, the LSTM model and Phased LSTM (PLSTM) model were used to predict the following features of gait one by one. For every model the input of data is one of the featuresat time $t$, the output of data is this feature at the time $t+1$. So, the input unit is one, the output unit is one. The LSTM network is created based on the keras framework.

The features of the gait are the characteristic data of human lumbar posture collected by sensors, which are (1) the acceleration of $\mathrm{x}, \mathrm{y}$ and $\mathrm{z}$ axes in terms of $(\mathrm{g})$, which are ax, ay and az; (2) the angular acceleration of $x, y$ and $z$ axes in terms of (deg/s), which are wx, wy and wz; (3) the angular acceleration of $\mathrm{x}, \mathrm{y}$ and $\mathrm{z}$ axes in terms of (deg), which are anglex, angley and anglez.

For multi-feature gait prediction, the input of data is as following features at time t, the outputs of data are thosefeatures at the time $t+1$. So, the input units are three, the output units are three. The LSTM network is created based on the keras framework.

The data used in multi-feature gait prediction experiments are the acceleration of $\mathrm{x}, \mathrm{y}$ and $\mathrm{z}$ axes, the angular acceleration of $\mathrm{x}, \mathrm{y}$ and $\mathrm{z}$ axes, the angular acceleration of $\mathrm{x}, \mathrm{y}$ and $\mathrm{z}$ axes, and the angular acceleration of $\mathrm{x}, \mathrm{y}$ and $\mathrm{z}$ axes. The three matrices are [ax ay az], [wx wy wz], [anglex angley anglez]. The three matrices are respectively used as input variables for prediction.

The process of model establishment is shown in Figure 3.

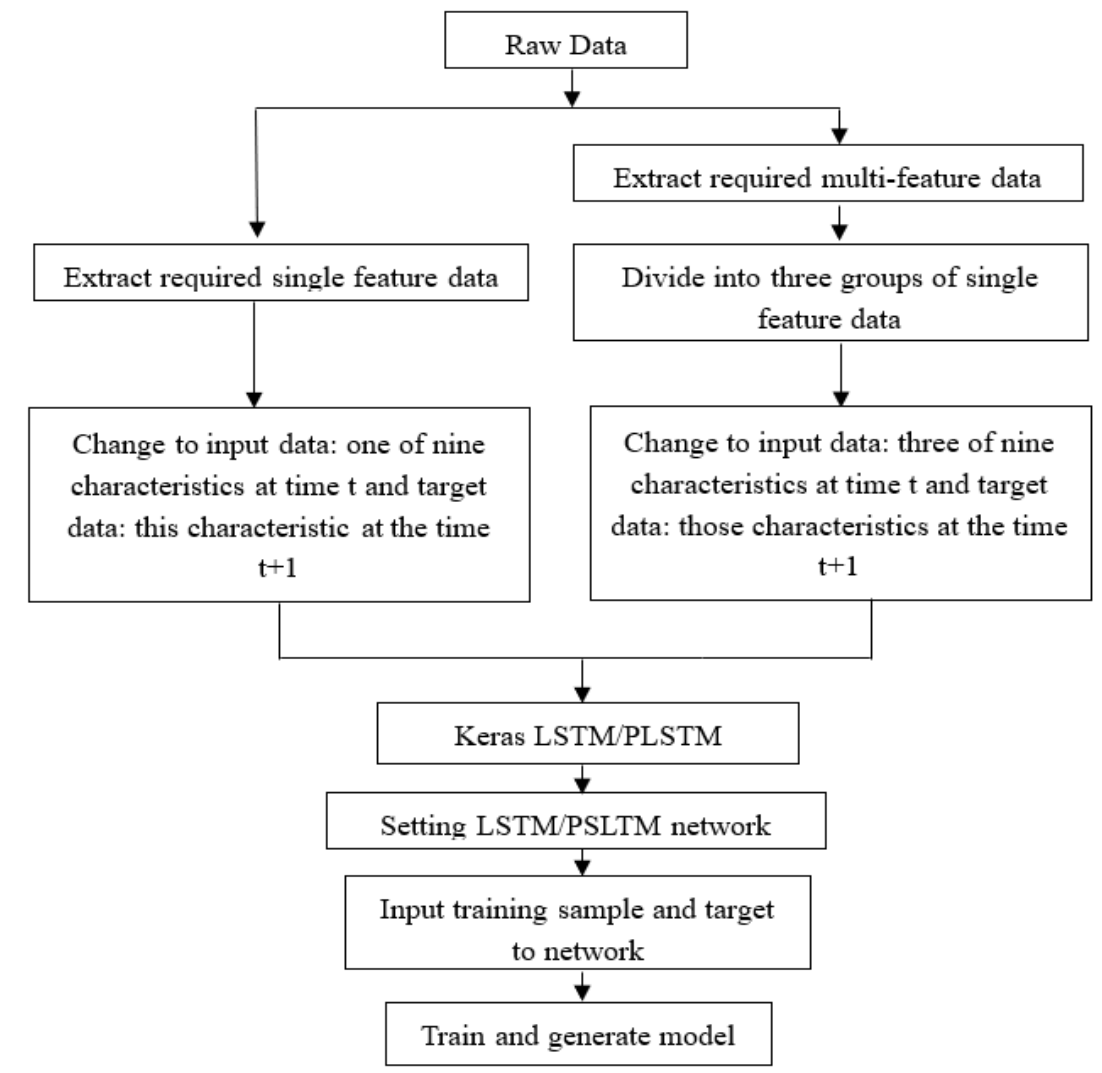

Figure 3. The flowchart of model establishment 


\section{EXPERIMENTS}

The experimental data is collected by the BWT901 nine-axis acceleration gyro angle sensor. The acquisition process is as follows:

(1) Connect the sensor to the PC through USB-HID. After the connection is successful, open the data receiving software Mini IMU on the PC side for data recording, as shown in Figure 4.

(2) When collecting data, fix the sensor to the waist of the human body, collect data for walking, walking and sitting, and record the data through software, as shown in Figure 5.

(3) In order to record the human gait data more smoothly, at the time of recording, each time after the end of the action, the stagnation time is used as the buffer time, and then the buffer time data will be cut off as the final data input. See figure 6for an example of experimental data.

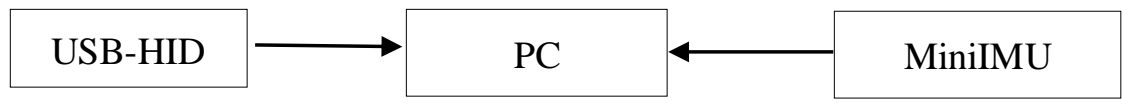

Figure 4. the connection between sensor and pc

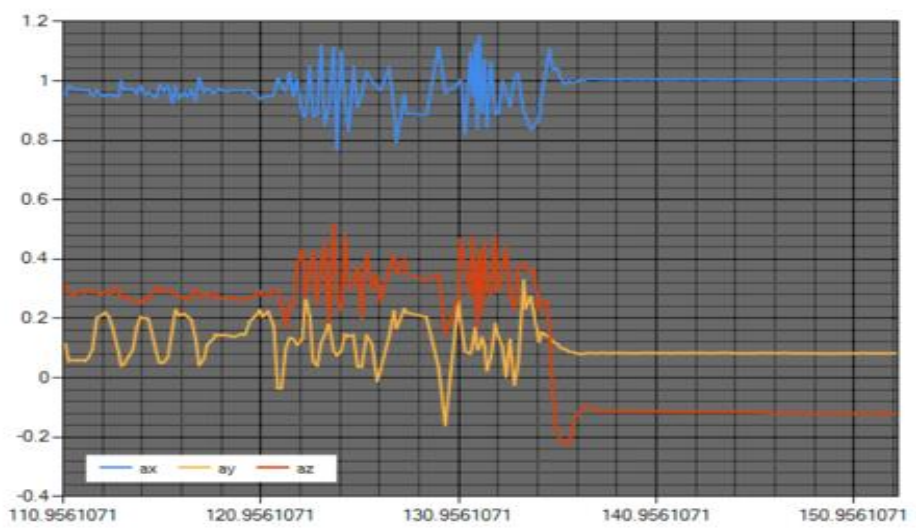

Figure 5. The UI of Recording software

\begin{tabular}{|c|c|c|c|c|c|c|c|c|c|} 
Time $(\mathrm{s})$ & $\mathrm{ax}(\mathrm{g})$ & $\mathrm{ay}(\mathrm{g})$ & $\mathrm{az}(\mathrm{g})$ & $\mathrm{wx}(\mathrm{deg} / \mathrm{s})$ & $w y(\mathrm{deg} / \mathrm{s})$ & $\mathrm{wz}(\mathrm{deg} / \mathrm{s})$ & AngleX $(\mathrm{deg})$ & AngleY $(\mathrm{deg})$ & AngleZ(deg) \\
\hline 68.432 & 0.0435 & -0.9819 & -0.1401 & -1.4648 & -0.1221 & -0.061 & 105.1392 & 60.2271 & -153.0945 \\
\hline 68.633 & 0.0425 & -0.9819 & -0.1406 & -1.77 & -0.061 & -0.1221 & 104.892 & 60.2545 & -153.1934 \\
\hline 68.842 & 0.0449 & -0.98 & -0.1406 & -1.5869 & -0.061 & -0.1221 & 104.6777 & 60.282 & -153.2538 \\
\hline 69.066 & 0.0454 & -0.9785 & -0.1436 & -1.5869 & -0.061 & 0.061 & 104.5294 & 60.2985 & -153.2593 \\
\hline 69.232 & 0.0415 & -0.978 & -0.1465 & -2.7466 & 0.4883 & 0.4883 & 104.3866 & 60.26 & -153.2043 \\
\hline 69.45 & 0.0356 & -0.9829 & -0.1597 & -3.479 & 1.5869 & 0.061 & 104.2877 & 60.1776 & -152.9517 \\
\hline 69.64 & 0.0527 & -0.9839 & -0.1802 & -2.5635 & 0.1831 & -1.0986 & 104.1229 & 60.1996 & -152.7209 \\
\hline
\end{tabular}

Figure 6. Example of experimental data

There are two part of experiments: collection of data and prediction experiment. The prediction experiment is divided into two parts. The first part is a single variable prediction experiment, and the second part is a multi-variable prediction experiment. 
International Journal of Artificial Intelligence and Applications (IJAIA), Vol.10, No.4, July 2019

In data collection experiment, we use the sensor which collects a value every 0.2 seconds to collect 365 s time series data totalling 1825 values. The $67 \%$ of them are used to train the model, and the rest are used to test.

Inprediction experiment, we create LSTM neural network with one input layer, one hidden layer, and one input layer. The hidden layer has 4 neurons, the hidden layer uses the sigmoid activation function, the number of iterations is 100 , the batch size is 1 and the loss function is mean square error, the optimization algorithm uses the adam algorithm. We use both LSTM and PLSTM to build models for gait prediction. The models establishment are following the steps in section 3 . Thepredict results of LSTM model were shown in Figure 7 and Figure 8. Thepredict results of PLSTM model were shown in Figure 9 and Figure 10.

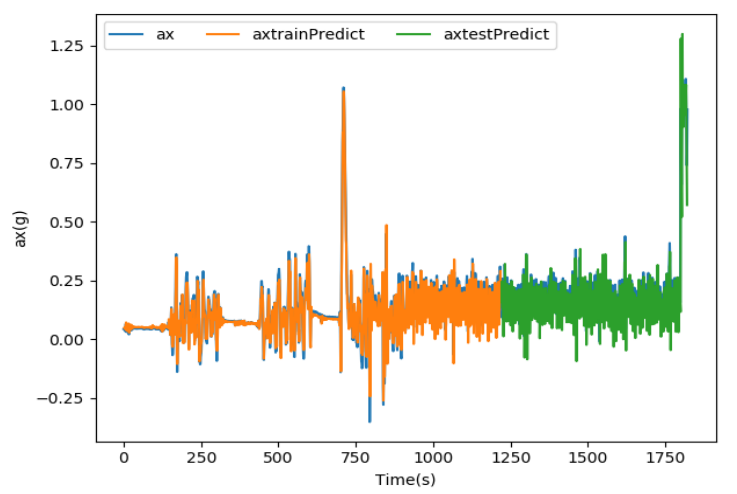

Figure 7. LSTM-example of univariate prediction experiment

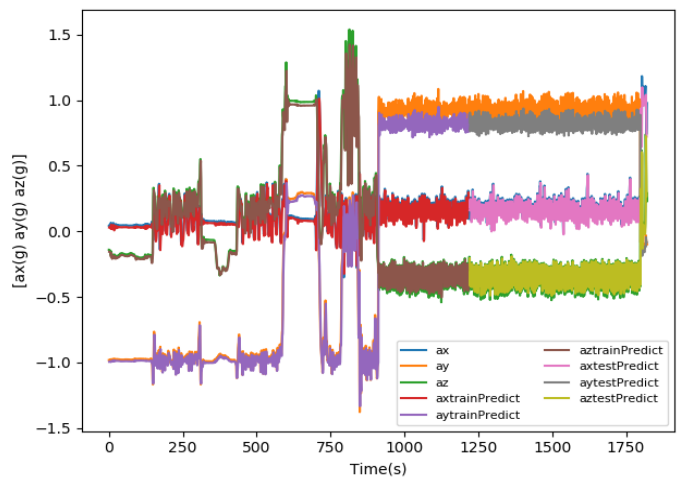

Figure 8. LSTM-example of multivariate prediction experiments

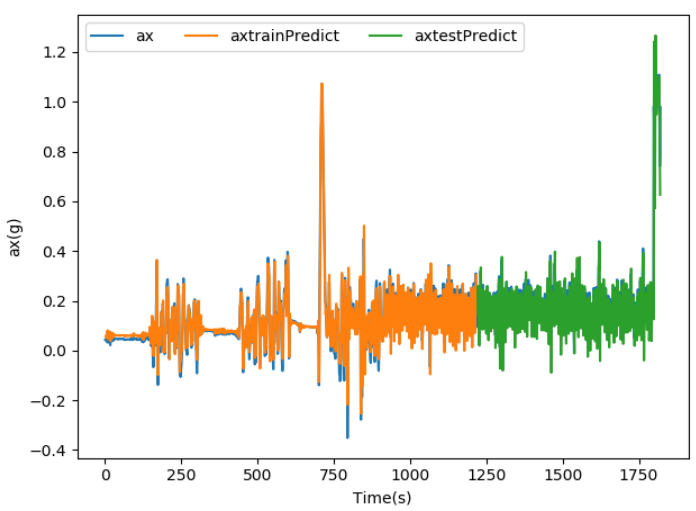


Figure 9. PLSTM-example of univariate prediction experiment

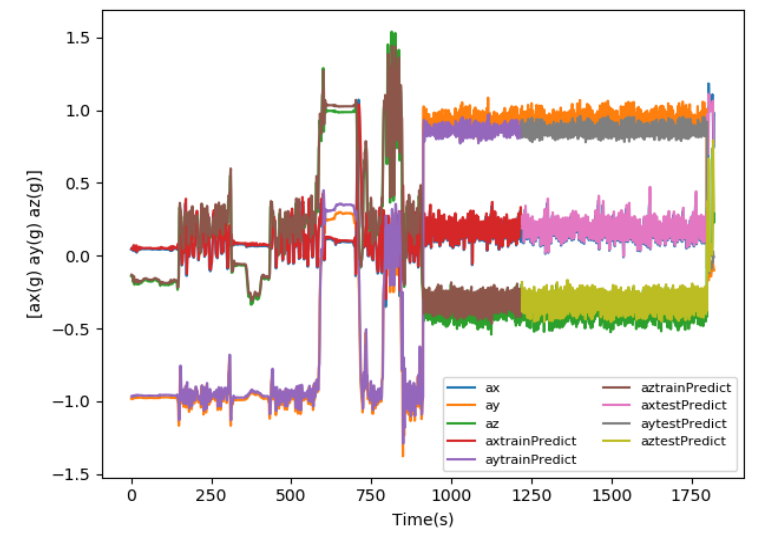

Figure 10. PLSTM-example of univariate prediction experiment

The RMSE of LSTM model and PLSTM model predicted results corresponding to univariate input is shown in Table 1 and Table 3,the RMSE of LSTM model and PLSTM model predicted results corresponding to multivariate input is shown in Table 2 and Table 4.The processing speed of the LSTM model and the PLSTM model is shown in Table 5.

Table 1. Predicted results of LSTM model corresponding to univariate input

\begin{tabular}{|l|l|l|l|l|l|l|l|l|l|}
\hline $\begin{array}{c}\text { input } \\
\text { RMSE }\end{array}$ & ax & ay & az & wx & wy & wz & anglex & angley & anglez \\
\hline Train & 0.05 & 0.08 & 0.08 & 7.72 & 12.87 & 8.45 & 22.47 & 2.69 & 23.03 \\
\hline Test & 0.08 & 0.09 & 0.12 & 8.83 & 19.43 & 14.89 & 6.93 & 3.77 & 32.83 \\
\hline
\end{tabular}

Table 2. Predicted results of LSTM model corresponding to multivariate input

\begin{tabular}{|l|l|l|l|}
\hline \multicolumn{1}{|c|}{ input } & [ax ay az $]$ & [wx wy wz $]$ & [anglex angley anglez $]$ \\
& & & \\
\hline Train & 0.04 & 0.05 & 0.07 \\
\hline Test & 0.05 & 0.07 & 0.06 \\
\hline
\end{tabular}

Table 3. Predicted results of PLSTM model corresponding to univariate input

\begin{tabular}{|l|l|l|l|l|l|l|l|l|l|}
\hline $\begin{array}{c}\text { input } \\
\text { RMSE }\end{array}$ & ax & ay & az & wx & wy & wz & anglex & angley & anglez \\
\hline Train & 0.05 & 0.08 & 0.07 & 7.82 & 12.67 & 8.36 & 22.32 & 3.37 & 22.57 \\
\hline Test & 0.08 & 0.08 & 0.11 & 8.92 & 19.23 & 15.13 & 6.90 & 4.17 & 31.60 \\
\hline
\end{tabular}

Table 4. Predicted results of PLSTM model corresponding to multivariate input

\begin{tabular}{|l|l|l|l|}
\hline RMSE & input & [ax ay az $]$ & [anglex angley anglez $]$ \\
\hline
\end{tabular}


International Journal of Artificial Intelligence and Applications (IJAIA), Vol.10, No.4, July 2019

\begin{tabular}{|l|l|l|l|}
\hline Train & 0.04 & 0.07 & 0.09 \\
\hline Test & 0.05 & 0.08 & 0.07 \\
\hline
\end{tabular}

Table 5. Training time of the LSTM model and the PLSTM model

\begin{tabular}{|l|l|l|}
\hline $\begin{array}{c}\text { model } \\
\text { time(s) }\end{array}$ & LSTM & PLSTM \\
\hline univariate input & 202 & 200 \\
\hline multivariate input & 599 & 490 \\
\hline
\end{tabular}

The predicted result score is determined by RMSE. The RMSE stands for the distance between the predicted result and the original data, it means that the distance is shorter, the result is better. From the data in the table, it can be seen that the prediction result of single variable input is better than that of $\mathrm{x}, \mathrm{y}$ and $\mathrm{z}$ axis acceleration, while that of multi-variable input is better. As a whole, LSTM network has ideal prediction results and good prediction results for different human postures. Therefore, it is of practical significance to use LSTM to predict gait sequence to predict falls. From the scores of LSTM and PLSTM, it can be seen that PLSTM model is better than LSTM model in terms of univariate prediction, but little worse than LSTM model in terms of multivariate prediction. The reason for this result is that PLSTM network is more good at modelling for the sequence data with inconsistent sampling periods. But the data trained by the experiment is the sequence data with the same sampling period. Therefore, because of the time gate, some data is not updated, resulting in a lower accuracy. But it can be seen PLSTM model is truly faster than LSTM model.

\section{Conclusions}

Experiments show that the prediction results of multi-variable input are better than that of singlevariable input, which indicates that human gait or posture needs three-dimensional data to predict or detect better. According to comparing between LSTM and PLSTM, we find that PLSTM can accelerate RNN model, and PLSTM is more suitable for training sequence data with inconsistent sampling periods, and LSTM network is better at training sequence data with consistent sampling periods.However, there are still some shortcomings in this paper. Firstly, when estimating, modelling and forecasting gait data, the collected data types are relatively single, and various gait types should be collected to predict. This analysis from many aspects can improve the accuracy and feasibility of gait forecasting analysis and is more efficient and effective in practical application. Secondly, the gait sequence with very short time interval is analysed in this paper, and the gait span in real situation is not considered. At the same time, this paper only comparing LSTM and its variant of prediction, without comparing other neural network model, which fails to reflect the superiority of LSTM method very well. Moreover, I have not applied this method further.

The next research includes the following aspects: (1) Increasing the number of layers of neural networks and combining two or more kinds of neural networks to optimize the network structure [13] and improve the accuracy of prediction. (2) Combining gait prediction with gait recognition to enhance the practicability of the model.

\section{ACKNOWLEDGEMENTS}

This work is supported by National Science Foundation of China under Grants 51711012, and Beijing Municipal Education Commission under grant KM201811232016. 


\section{REFERENCES}

[1] Scheffer A C, Schuurmans M J, Van Dijk N, et al. Fear of falling: measurement strategy, prevalence, risk factors and consequences among older persons[J]. Age \& Ageing, 2008, 37(1): 19-24.

[2] Zhou Chuang; Fu Jia-yu; Lei Zhong-gui; Wang Zhi-xiong.Current status and prospects in the eldly fall detection research [J]. COMPUTER ENGINEERING \& SOFTWARE,2018,v.39;No.462,119123.

[3] Wu G. Distinguishing fall activities from normal activities by velocity characteristics[J]. Journal of Biomechanics, 2000, 33(11): 1497-500.

[4] Bourke A K, O'Donovan K J, Olaighin G. The identification of vertical velocity profiles using an inertial sensor to investigate pre-impact detection of falls[J]. Medical Engineering \& Physics, 2008, 30(7): 937.

[5] Nyan M N, Tay F E, Mah M Z. Application of motion analysis system in preimpact fall detection[J]. Journal of Biomechanics, 2008, 41(10): 2297-2304.

[6] Lina Tong. Human fall recognition method based on mechanical information acquisition system[D]. University of Science and Technology of China, 2011.

[7] Shi G, Zhang J, Dong C, et al. Fall detection system based on inertial mems sensors: Analysis design and realization[C]// IEEE International Conference on Cyber Technology in Automation, Control, and Intelligent Systems. IEEE 2015: 1834-1839.

[8] CHEN Chaoqiang, JIANG Lei, WANG Heng. Gait prediction method of lower extremity exoskeleton based on SAE and LSTM neural network[J]. Computer Engineering and Applications:1-11[2019-0224].

[9] ZENG M,NGUYEN L T,YU B,et al. Convolutional neural networks for human activity recognition using mobile sensors [C]//2014 6th International Conference on MobileComputing, Applications and Services (MobiCASE).USA: IEEE,2014:197-205

[10] XU Fan, CHENG Hua, FANG Yi-quan. A gait pattern classification method based on CLSTM [J]. Journal of East China University of Science and Technology (Natural Science Edition)2017,43(04):553-558.

[11] Yu Liu;Shuting Dong;Mingming Lu;Jianxin Wang.LSTM Based Reserve Prediction for Bank Outlets[J].Tsinghua Science and Technology,2019,v.24,79-87.

[12] Daniel Neil; Michael Pfeiffer; Shih-ChiiLiu. Phased LSTM: Accelerating Recurrent Network Training for Long or Event-based Sequences [C]//arXiv:1610.09513v1 [cs.LG] 29 Oct 2016

[13] Kumar, A., Sang wan, S. R., Arora, A., Nayyar, A., \& Abdel-Basset, M. (2019). Sarcasm Detection Using Soft Attention-Based Bidirectional Long Short-Term Memory Model with Convolution Network. IEEE Access. 


\section{Authors}

Qili Chen was born in YuXian, ShanXi Province, China in 1985. She received the B.S degrees in automation from Chongqing University of Posts and Telecommunications in 2007, M.S and Ph.D. degrees in pattern recognition and intelligent system from Beijing University of Technology in 2010 and 2014. From Sep. 2012 to Aug.2013, she visited University of Wisconsin, Milwaukee (USA) in Department of Mathematical Sciences. Since 2014, She has been a Lecture with Automation College, Beijing Information Science and Technology University. Her research interests include deep neural network, Modeling and optimal control.

Bofan liang was born in WuZhou, Guangxi Zhuang Autonomous Region, china in 1997.He is an undergraduate at Beijing Information Science and Technology University.
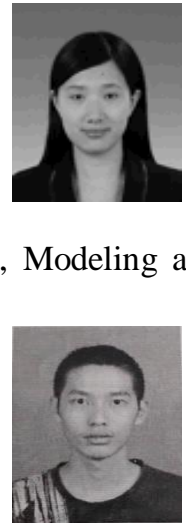